\title{
Formulation of Herbal Capsule Containing Trigonella Foenum-Graecum Seed Extract for the Treatment of Diabetes
}

\author{
Divya Jyothi ${ }^{*}{ }_{1}$, Marina Koland ${ }^{2}$, Sneh Priya ${ }^{2}$, Jainey Puthenveetil James ${ }^{3}$ \\ 'Department of Pharmacognosy, Nitte Gulabi Shetty Memorial Institute of Pharmaceutical Sciences, Nitte University, Deralakatte Karnataka, INDIA. \\ 2Department of Pharmaceutics, Nitte Gulabi Shetty Memorial Institute of Pharmaceutical Sciences, Nitte University Deralakatte, Karnataka, INDIA. \\ ${ }^{3}$ Department of Pharmaceutical Chemistry, Nitte Gulabi Shetty Memorial Institute of Pharmaceutical Sciences, Nitte University Deralakatte, Karnataka, INDIA.
}

\begin{abstract}
Background: Trigonella foenum-graecum (fenugreek) seeds are reported to contain multiple antidiabetic constituents and hence widely used for the treatment of diabetes mellitus. The present investigation was aimed to formulate capsule formulations containing crude extract of fenugreek seeds in order to obtain antidiabetic formulations with more effective oral hypoglycemic activity, less side effects, increased patient compliance thereby providing multifaceted benefits. Methodology: Capsule formulations were prepared by encapsulation of granules prepared from the fenugreek seed extract with various concentration of sodium starch glycolate as superdisintegrant (0-5\%). Finished capsule formulations were evaluated for weight variation, disintegration time, drug content (trigonelline) content, in vitro-drug release, in vivo antidiabetic activity studies. Results: Fenugreek capsule formulations pass the test for weight since the percentage deviation of individual weight of capsule from mean were found within $\pm 7.5 \%$. Drug (trigonelline) content of all the formulations were more than $85 \%$. Disintegration time ranged from 7-15 mins. Dissolution profile showed 77.06 $-90 \%$ drug release in phosphate buffer of pH 6.8 after 6 hours. Antidiabetic activity studies of capsules significantly $(p \leq 0.001)$ reduced blood glucose level in diabetic rats after 15 days of treatment when compared to diabetic control group. Conclusion: From the results, we concluded that formulation
\end{abstract}

of fenugreek seed extracts into suitable and appropriate herbal dosage form may be more desirable, advantageous and therapeutically more beneficial than incorporating the direct plant materials for the treatment of diabetes.

Key words: Diabetes Mellitus, Fenugreek, Trigonelline, Capsule, Superdisintegrants.

Key messages: Fenugreek is reported to have antidiabetic effect and various formulations are reported from either from fenugreek powder or isolated compounds. But this study reports simple formulation prepared from extract of fenugreek with improved antidiabetic effect.

Correspondence :

Divya Jyothi, Department of Pharmacognosy, Nitte Gulabi Shetty Memorial Institute of Pharmaceutical Sciences, Nitte University, Deralakatte, Karnataka, INDIA.

Phone: +918722821164

Email: divya.jyothi84@gmail.com

DOI: 10.5530/jyp.2017.9.70

\section{INTRODUCTION}

Diabetes mellitus, commonly known as diabetes, is one of the world's oldest known diseases. WHO estimated in 1995 that the number of people with diabetes in the world would reach 300 million by 2025 . At present, the treatment of diabetes mainly involves a sustained reduction in hyperglycemia by the use of biguanides, thiazolidinediones, sulphonylureas, D-phenylalanine derivatives, meglitinides and a-glucosidase inhibitors in addition to insulin. Despite considerable progress in the treatment of diabetes search for newer drugs continues as the existing synthetic drugs fails to maintain euglycaemia, controlling long-term microvascular, macrovascular complications and provide economic burden particularly to the rural population across the globe. ${ }^{1}$ Thus, alternative therapy has gained the attention to shift the paradigm towards indigenous plant and herbal formulations with antidiabetic potential.

Diabetic mellitus (DM) is a widely spread epidemic disease that results from the absence of insulin, decreased secretion and/or impaired function. Since Diabetic mellitus (DM) is a multifactorial disease, the available pharmaceuticals, despite their sensible treatment, target mostly one pathway to control hyperglycemia and encounter several side effects. Therefore new therapeutic paradigms aim to hit several pathways using only one agent. ${ }^{2}$ Traditionally, antidiabetic plants and /or their active constituents may fulfill this need and one of them is Fenugreek.
Trigonella foenum-graecum (Fenugreek) belonging to the family Leguminosae is one of the oldest medicinal plants, dating back to the ancient Egyptians, Greeks and Romans, who used it as a culinary and medicinal herb. The hypoglycaemic effect of fenugreek seeds has been granted to the presence of alkaloid trigonelline, ${ }^{3}$ an insulin-stimulating substance 4-hydroxyisoleucine, ${ }^{4,5}$ soluble fibre, ${ }^{6}$ coumarin, ${ }^{7}$ scopoletin and fenugreekine ${ }^{8}$ which are said to act by several mechanisms. By considering above facts, present study is aimed at developing formulations from crude plant extract of fenugreek containing several antidiabetic constituents which act by several modes of action to influence multiple biological pathways and thereby producing more effective oral hypoglycemic activity. The study was also designed to produce formulation which are safe, cheaper and which can also alleviate the diabetic symptoms thereby providing multifaceted benefits.

\section{MATERIALS AND METHODS}

Materials

Lactose, Magnesium stearate, talc, starch, Sodium starch glycolate, Streptozotocin was procured from Hi Media Ltd, Mumbai. Glibenclamide a standard anti-diabetic drug was purchased from local market. Standard trigonelline hydrochloride was obtained from natural remedies, Bangalore. All the chemicals and reagents used in this study were of analytical grade.

This is an open access article distributed under the terms of the Creative Commons Attribution-NonCommercial-ShareAlike 4.0 License, which allows others to remix, tweak, and build upon the work non-commercially, as long as the author is credited and the new creations are licensed under the identical terms. 


\section{Extraction of fenugreek seeds and preliminary phytochemical analysis of the extract}

Trigonella foenum graecum (Fenugreek) seeds were purchased from local market which was authenticated by Prof (Dr) Nagalakhmamma St. Aloysius College, Mangalore. Seeds were, pulverized, sieved through 40 mesh to obtain coarse powder. ${ }^{9}$

Powdered fenugreek seeds were extracted in batches of $500 \mathrm{~g}$ each with $2.5 \mathrm{~L}$ of ethanol (95\%) using soxhlet extractor for 2 days. After the extraction period, solutions were filtered and concentrated by rotary vacuum evaporator. Extract was subjected to different chemical tests for the detection of various phytoconstituents.

\section{Estimation of trigonelline by UV-spectrophotometric method}

Trigonelline constitutes important antidiabetic constituent present in fenugreek, hence estimation of trigonelline content in fenugreek extract were done.

Ultraviolet spectrophotometric method was carried out using phosphate buffer ( $\mathrm{pH}$ 6.8) as solvent media and trigonelline hydrochloride as standard as per reported method with slight modification. ${ }^{10}$ Standard trigonelline hydrochloride and fenugreek extract solution in phosphate buffer ( $\mathrm{pH}$ 6.8) was scanned between UV-Visible range for obtaining absorbance maxima. Then the absorbance of the different serial diluted samples of standard was measured at the $\lambda$ max using a UV spectrophotometer (V-630, Jasco) and a standard calibration curve was plotted with concentration against absorbance.

\section{Preparation of fenugreek granules}

Fenugreek granules were prepared by wet granulation method. Viscous sticky extract was mixed with lactose till it produces coherent mass. No binding agents are added externally since mucilaginous constituents present in fenugreek act as binding agent. Then required quantity of starch powder (5\%) was added and then powder blend was passed through \# 12 to produce granules. Granules were gently spread and dried at temperature below $60^{\circ} \mathrm{C}$. Dry granules were weighed and their weight was recorded. Further dry granules were regranulated by passing through \# 16 placed on oversize \# 44 to get uniform sized granules. Granules retained on \# 44 were collected and weighed. Fines which passed through \# 44 were also weighed. Fines equivalent to $15 \%$ of the weight of granules were mixed with granules and other ingredients such as starch powder, talc and magnesium stearate were added in required quantities.

Granules were also prepared containing sodium starch glycollate (SSG) as superdisintegrant. After addition of lactose to the extract, SSG were incorporated at different concentration $(2 \%, 3 \%, 5 \%)$ separately and granulations were carried out similar way as described above.

\section{Evaluation of fenugreek granules}

Prepared fenugreek granules were subjected for determination of bulk density, tapped density, Hausner ratio, Carr's index, angle of repose and bulkiness in order to assess the flow property of granules. ${ }^{11,12}$

\section{Formulation of fenugreek capsules}

Prepared granules were packed into hard gelatin capsule (size 0) using hand operated capsule filling machine such that each capsule contains $1000 \mathrm{mg}$ of granules. Fenugreek capsules without SSG were labelled as F1 and capsules containing $2 \%, 3 \%$ and $5 \%$ of sodium starch glycolate (SSG) were labelled as F2, F3, and F4 respectively and list of capsule ingredients are shown in Table 1.

\section{Evaluation of fenugreek capsules ${ }^{13}$ Estimation of drug content (trigonelline) in capsules}

Granules from 10 capsules were mixed and weight of powder equivalent to $12.55 \mathrm{mg}$ of trigonelline and extracted with the phosphate buffer of $\mathrm{pH}$ 6.8 for $30 \mathrm{~min}$. These solutions were filtered, suitably diluted and absorbance was measured at $265 \mathrm{~nm}$ against blank solution (phosphate buffer pH 6.8) using a UV spectrophotometer.

\section{Determination of uniformity of weight}

Twenty capsules were selected. Each capsule was weighed on an analytical balance, carefully emptied of its content, the shells reweighed and the weight of content determined. The collective weight of content, average weight of content per capsule and the deviations (\%) of individual content weights from the mean were calculated.

\section{Determination of disintegration time}

Disintegration times for capsules were determined by disintegration apparatus. Six capsules were placed in six tubes of the basket and the apparatus was operated using water as release medium maintained at $37 \pm 2^{\circ} \mathrm{C}$. The capsules were observed and the times taken for complete disintegration of all capsules were determined.

\section{In vitro dissolution study of capsules}

In vitro dissolution study of all the prepared capsule formulations was done using USP Type II paddle dissolution apparatus (Electrolab USP dissolution tester TDT-08L) using $900 \mathrm{ml}$ phosphate buffer $\mathrm{pH} 6.8$ at 100 $\mathrm{rpm}$ and results were compared with drug release of trigonelline from capsule formulation containing fenugreek seed powder which is labelled as F0. An aliquot amount of the sample was withdrawn at regular time intervals and the same volume of pre-warmed $\left(37 \pm 0.5^{\circ} \mathrm{C}\right)$ fresh dissolution medium was replaced. The samples were filtered, suitably diluted and Trigonelline in each sample was analyzed by using Shimadzu UV-spectrophotometer at $265 \mathrm{~nm}$.

\section{Investigation of in vivo antidiabetic activity of oral dosage forms}

Albino Wister rats of both sexes weighing 150-200 g were used. They were obtained from the central animal house KSHEMA Deralakatte, Mangalore, India. The animals were maintained under controlled conditions of temperature $\left(22 \pm 2^{\circ} \mathrm{C}\right)$, humidity $(50 \pm 5 \%)$ and $12 \mathrm{~h}$ lightdark cycles. They were fed commercial stock diet and water ad libitum. The animals were housed individually in sanitized polypropylene cages containing sterile paddy husk as bedding. Animals were acclimatized to laboratory conditions prior to experimental protocol.

A method reported by Rakieten et al (1963) for screening anti diabetic activity by using antibiotic streptozotocin was followed. ${ }^{14,15}$ Experimental diabetes was induced in overnight fasted rats by single intraperitoneal injection of streptozotocin $(60 \mathrm{mg} / \mathrm{kg} \mathrm{b.w})$ dissolved in freshly prepared $0.1 \mathrm{M}$ citrate buffer ( $\mathrm{pH} 4.5$ ). After 3days, fasting blood glucose levels were measured and the animals showing fasting blood glucose level $>250$ $\mathrm{mg} / \mathrm{dl}$ were selected for further experimentation. ${ }^{16}$

In the present study, adult male albino wister rats weighing 150-200 g, 5-6 weeks were selected. They were divided into 6 groups of 6 animals each.

Group 1: Normal non-diabetic control rats.

Group 2: Diabetic (STZ) control

Group 3: Diabetic rats treated with Glibenclamide $(0.5 \mathrm{mg} / \mathrm{kg} \mathrm{b.w})$ orally Group 4: Diabetic rats treated with fenugreek Capsule F1

(Dose equivalent to $1 \mathrm{~g} / \mathrm{kg}$ of extract)

Group 5: Diabetic rats treated with fenugreek Capsule F4 
(Dose equivalent $1 \mathrm{~g} / \mathrm{kg}$ of extract)

Group 6: Diabetic rats treated with fenugreek Capsule F0

Dose of the fenugreek formulations is selected based on the study reported by Gupta A .et al which states that effective dose of fenugreek for diabetes is $1 \mathrm{~g}$ of a hydroalcoholic extract. ${ }^{17}$ Fasting blood glucose (FBG) level of each rat was measured on 0,5 th, 10 th and 15 th day by using a one touch glucometer (Accucheck). All the data were analysed by one-way analysis of variance (ANOVA) followed by Dunnett's test.

\section{RESULTS AND DISCUSSION}

\section{Extraction of fenugreek seeds and preliminary phytochemical analysis of the extract}

Yield of the fenugreek extracts obtained was $12 \%$. The preliminary phytochemical analysis of fenugreek extracts showed the presence of alkaloids, phenols, flavonoids, tannins, steroids, carbohydrates, proteins and amino acids.

\section{Estimation of Trigonelline by UV-spectrophotometric method}

Amount of trigonelline in fenugreek extracts were estimated by UV-spectrophotometric method. UV spectrum of standard trigonelline solution showed maximum absorption at wavelength of $265 \mathrm{~nm}$ and hence $\lambda$ max of $265 \mathrm{~nm}$ was selected and utilized for the estimation of trigonelline. UV spectrum of fenugreek extract also showed the peak for trigonelline at wavelength of $265 \mathrm{~nm}$ which showed that absorption peak of trigonelline in fenugreek extract match with the absorption peak of standard trigonelline.

For preparation of standard plot, different concentration of standard trigonelline solution was prepared in phosphate buffer of $\mathrm{pH}(6.8)$ and absorbance was measured at $265 \mathrm{~nm}$. Trigonelline showed good linearity with Regression coefficient 0.998 at the concentration range of $6-18 \mu \mathrm{g} / \mathrm{ml}$ which indicated that Beer's law was obeyed in this concentration range (Figure 1). Amount of trigonelline in fenugreek extract was calculated from calibration curve equation $Y=0.023 x+0.001$ which was found to be $250 \mathrm{mg} / \mathrm{g}$ of the extract.

\section{Preparation and evaluation of granules of fenugreek}

Fenugreek granules were prepared from extract using various excipients containing different concentration of superdisintegrants by wet granulation method and prepared granules were subjected to preformulation studies and results of which is shown in Table 2.

There was no significant difference in bulk densities, tapped densities of prepared granules. Hausner ratio was found in the range of 1.07-1.18.
Since Hausner ratios were below 1.24 indicates good flow property of granules. Granules of almost all the formulations gave a compressibility index ranged from $6.57-15.85 \%$ and angle of repose in the range of $22.2^{\circ}-30.4^{\circ}$. A compressibility index of greater than $25 \%$ is considered to be an indication of poor flowability and below $15 \%$ an indication of excellent flowability of granules. Since all the formulations had a Carr's index below $15 \%$ and angle of repose below $30^{\circ}$ granules possess good flow property.

\section{Formulation and evaluation of fenugreek capsule}

All capsule formulations were subjected to various evaluations and results of which are shown in Table 3.

Fenugreek capsule formulations pass the test for weight variation according to I.P 2010 since the percentage deviation of individual weight of capsule from mean were found within $\pm 7.5 \%$. Drug (trigonelline) content of all the capsule formulations were more than $85 \%$. Disintegration time of formulations F1, F2, F3, F4 was found to be 15, 10, 9, 7 mins respectively which indicate that as the concentration of SSG increased the rate of disintegration also increased.

\section{In vitro dissolution study of capsules}

Rate and extent of release of trigonelline from capsule formulations were determined by carrying out in vitro dissolution study and results are shown in graphical representation in Figure 2.

Dissolution profile of fenugreek capsules indicated that the percentage release of trigonelline from formulation F1 was found to be $77.06 \pm 1.01$ after 6 hours. Since the release of trigonelline from F1 was incomplete, dissolution studies were further continued till $12 \mathrm{~h}$ to obtain drug release of $92 \%$. Slow and incomplete release of trigonelline from $\mathrm{F} 1$ is due to the presence of high percentage of mucilage (a natural gummy substance present in the coatings of seed) in fenugreek. Mucilage present in

\section{Table 1: Formulation of Capsules}

\begin{tabular}{ccccc}
\hline \multirow{2}{*}{ Ingredients } & \multicolumn{4}{c}{ Quantity/ capsule(mg) } \\
\cline { 2 - 5 } & F1 & F2 & F3 & F4 \\
\hline Fenugreek extract & 500 & 500 & 500 & 500 \\
Lactose monohydrate & 340 & 320 & 310 & 290 \\
Starch powder (10\%) & 100 & 100 & 100 & 100 \\
Talc (3\%) & 30 & 30 & 30 & 30 \\
Magnesium stearate (3\%) & 30 & 30 & 30 & 30 \\
Sodium Starch Glycolate & ----- & 20 & 30 & 50 \\
\hline
\end{tabular}

Table 2: Evaluation of fenugreek granules

\begin{tabular}{ccccccc}
\hline $\begin{array}{c}\text { Formulation } \\
\text { Code }\end{array}$ & $\begin{array}{c}\text { Bulk density } \\
(\mathrm{g} / \mathrm{ml})\end{array}$ & $\begin{array}{c}\text { Tapped } \\
\text { density }(\mathrm{g} / \mathrm{ml})\end{array}$ & $\begin{array}{c}\text { Hausner } \\
\text { ratio }\end{array}$ & $\begin{array}{c}\text { Carr's } \\
\text { index }(\%)\end{array}$ & $\begin{array}{c}\text { Angle of repose } \\
\left(\varnothing^{\circ}\right)\end{array}$ & Bulkiness \\
\cline { 2 - 7 } F1 & $0.71 \pm 0.02$ & $0.76 \pm 0.02$ & $1.07 \pm 0.06$ & $6.57 \pm 0.07$ & $30.4 \pm 0.05$ & $1.40 \pm 0.03$ \\
F2 & $0.73 \pm 0.03$ & $0.79 \pm 0.01$ & $1.08 \pm 0.02$ & $7.59 \pm 0.05$ & $22.2 \pm 0.01$ & $1.36 \pm 0.01$ \\
F3 & $0.70 \pm 0.01$ & $0.81 \pm 0.03$ & $1.15 \pm 0.01$ & $13.58 \pm 0.01$ & $23.3 \pm 0.05$ & $1.42 \pm 0.01$ \\
F4 & $0.69 \pm 0.02$ & $0.82 \pm 0.01$ & $1.18 \pm 0.01$ & $15.85 \pm 0.03$ & $23.5 \pm 0.03$ & $1.44 \pm 0.04$ \\
\hline
\end{tabular}

All values are expressed as mean $\pm S D, n=3$. 
fenugreek extract forms a viscous tacky mass when exposed to fluids due to which drug release from the granules becomes slower. Mucilage derived from the seeds of fenugreek were also reported as binding agent, suspending agent, gelling agent and release retardant material in solid dosage forms. ${ }^{18-21}$ Since there was no satisfactory drug release obtained from F1, in order to improve drug release incorporation of SSG as superdisintegrant in fenugreek capsules were done. To explore the effect of amount of superdisintegrants, three formulations F2, F3, F4 were prepared which contained $2 \%, 3 \%$ and $5 \%$ of sodium starch glycolate as a superdisintegrant, respectively. Dissolution profile of capsules F2, F3, F4 showed more than $90 \%$ drug release after $6 \mathrm{hrs}$. It is observed that with increasing the concentration of sodium starch glycolate, the rate and extent of drug release from the formulation F1, F2, F3 were also increased. Thus, the release characteristics were significantly influenced by the concentration of superdisintegrants used. SSG act as superdisintegrant by rapid absorption of water and swelling leading to an enormous increase in volume of granules which result in rapid and uniform disintegration. Dissolution profile of prepared capsule formulations were also compared with the drug release from capsule formulation prepared by direct encapsulation of fenugreek seed powder. Result indicated that extent of drug release from prepared capsule formulation containing fenugreek extract is more when compared to capsule containing fenugreek powder which showed only $50 \%$ drug release after 12 hrs. Poor, incomplete dissolution and extraction from the crude plant material within the capsules might be amongst the reasons for the low active content and poor release characteristics, compared to the formulations of its extract as in capsules prepared in this study.

\section{Investigation of antidiabetic activity of oral dosage forms of Fenugreek}

Antidiabetic activity of selected capsule formulations were investigated in streptozotocin induced diabetic rats and activity was compared with the capsules containing crude fenugreek seed powder (F0) which is given in Table 4.

In the present study, hyperglycemia was observed in rats after 3 days of STZ induction. Compared with the normal rats, STZ-diabetic rats showed more than 4-folds increase in blood glucose level. Antidiabetic activity studies of fenugreek formulations showed that Capsules F1, F4 significantly $(\mathrm{p} \leq 0.001)$ reduced blood glucose level in diabetic rats by $58.90 \%$ and $64.72 \%$ respectively after 15 days of treatment when compared to diabetic control group. Treatment with fenugreek formulations in STZ-induced diabetic rats started reducing fasting blood glucose levels after 5 days and made them completely normoglycemic after 15 days. Among the capsule formulations, capsule F4 formulation showed significant reduction of blood glucose level $(\mathrm{p} \leq 0.001)$ after 5 days of treatment

\section{Table 3: Physical characterization of fenugreek capsules}

\begin{tabular}{cccc}
\hline \multirow{2}{*}{$\begin{array}{c}\text { Cormulation } \\
\text { Code }\end{array}$} & \multicolumn{3}{c}{ Evaluation parameters } \\
\cline { 2 - 4 } & $\begin{array}{c}\text { Weight variation } \\
(\mathrm{mg})\end{array}$ & $\begin{array}{c}\text { Trigonelline } \\
\text { content (\%) }\end{array}$ & $\begin{array}{c}\text { Disintegration Test } \\
\text { (mins) }\end{array}$ \\
\hline F1 & $986 \pm 0.4$ & $87.80 \pm 0.1$ & $15 \pm 0.3$ \\
F2 & $982 \pm 0.3$ & $86.85 \pm 0.5$ & $10 \pm 0.1$ \\
F3 & $984 \pm 0.4$ & $87.41 \pm 0.3$ & $9 \pm 0.4$ \\
F4 & $980 \pm 0.2$ & $89.06 \pm 0.3$ & $7 \pm 0.4$ \\
\hline
\end{tabular}

All values are expressed as mean $\pm \mathrm{SD}, \mathrm{n}=3$

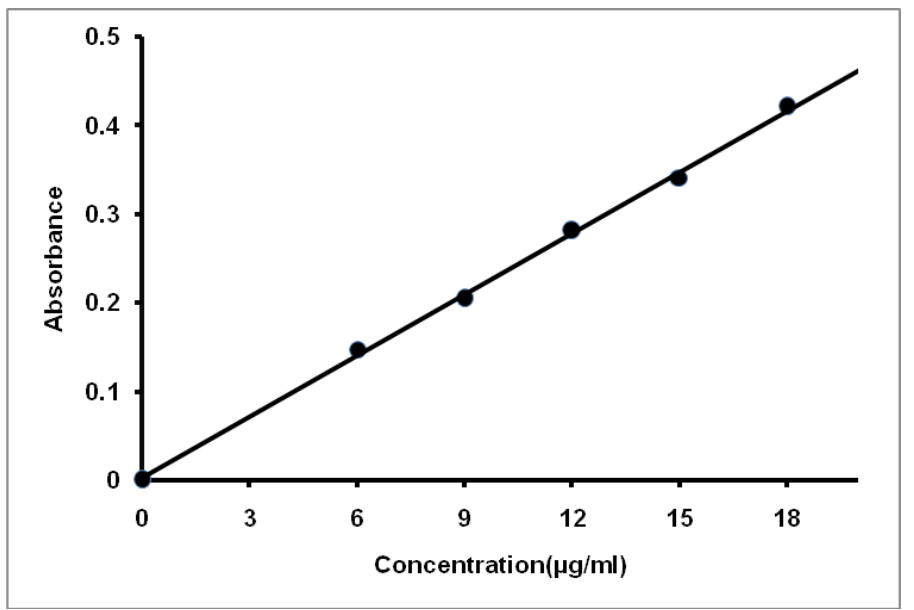

Figure 1: Standard plot of trigonelline hydrochloride in phosphate buffer at $265 \mathrm{~nm}$.

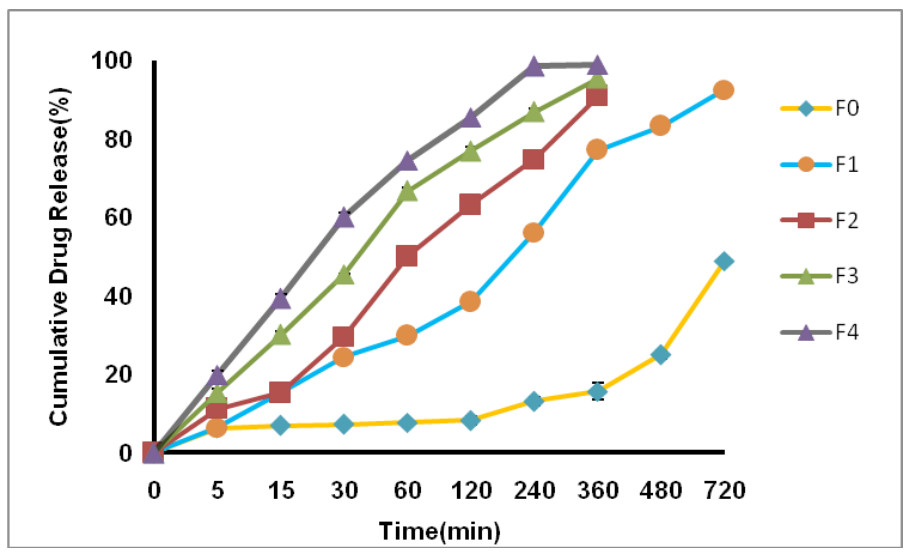

Figure 2: Release profile of trigonelline from capsules.

Table 4: Fasting blood sugar levels in rats receiving oral formulations

\begin{tabular}{cccccc}
\hline Groups & Treatment & \multicolumn{3}{c}{ Fasting blood sugar level in mg/dL } \\
\cline { 3 - 6 } & & Day 0 & Day 05 & Day 10 & Day 15 \\
\hline 1 & Normal Control & $76 \pm 0.3$ & $75 \pm 0.04$ & $74 \pm 0.4$ & $74 \pm 0.5$ \\
2 & Diabetic control & $278 \pm 0.5$ & $281 \pm 0.4$ & $286 \pm 0.4$ & $292 \pm 0.4$ \\
3 & Glibenclamide & $279 \pm 0.4$ & $96 \pm 0.4^{* * *}$ & $82 \pm 0.5^{* * *}$ & $79 \pm 0.7^{* * *}$ \\
4 & Capsules F1 & $278 \pm 0.4$ & $180 \pm 0.8^{* *}$ & $135 \pm 0.4^{* * *}$ & $120 \pm 0.4^{* * *}$ \\
5 & Capsules F4 & $278 \pm 0.3$ & $129 \pm 0.7^{* * *}$ & $122 \pm 0.8^{* * *}$ & $103 \pm 0.4^{* * *}$ \\
6 & Capsules F0 & $277 \pm 0.4$ & $210 \pm 0.6^{* *}$ & $172 \pm 0.5^{* *}$ & $140 \pm 0.6^{* * *}$ \\
\hline
\end{tabular}

Values are expressed as mean $\pm \mathrm{SEM} ; \mathrm{n}=06$ animals in each group; ${ }^{*} \mathrm{p} \leq 0.05,{ }^{* *} \mathrm{p} \leq 0.01,{ }^{* *} \mathrm{p} \leq 0.001$. 
than $\mathrm{F} 1$ and it was comparable to that the effect exerted by the reference drug, glibenclamide at the dose of $0.5 \mathrm{mg} / \mathrm{kg}$. Capsule formulation F4 was more effective as antidiabetic agent than F1 due to the presence of superdisintegrant in $\mathrm{F} 4$ which increases the rate of dissolution of granules thereby providing faster absorption of phytoconstituents hence producing quick onset of action. Rate of reduction of blood glucose from formulation F1 was found to be slow and it was continued till 15 days which may be due to the slow drug release from fenugreek extract and absence of superdisintegrant. Fenugreek extract contains mucilage which forms a viscous tacky mass when exposed to fluids due to which drug release from the granules becomes slower.

Capsule formulations containing fenugreek extract was found to be more effective as antidiabetic agent than capsule formulation containing crude fenugreek seed powder which showed only $52.05 \%$ reduction in blood sugar level after 15 days. Hence it is concluded that Capsule F4 formulations demonstrated effective anti-hyperglycemic activity in STZ induced diabetic rats; and restored normal glucose level.

\section{CONCLUSIONS}

All the prepared oral formulations containing fenugreek extract showed better antidiabetic activity when compared with formulation containing fenugreek seed powder. Present study strongly supports the assertions that the formulation of plant extracts into suitable and appropriate herbal dosage form may be more desirable, advantageous and therapeutically more beneficial than incorporating the direct plant materials/isolated phytoconstituents.

\section{ACKNOWLEDGEMENT}

I am thankful to Nitte University and NGSM institute of pharmaceutical sciences for providing all the facilities and requirement for carrying out this work. Also thankful to Natural Remedies, Bangalore for providing standard compound of Trigonelline.

\section{CONFLICT OF INTEREST}

No Conflict of interest.

\section{ABBREVIATION USED}

UV: Ultra Violet; SSG: Sodium Starch Glycollate; mg: milligram; nm: nanometer.

\section{REFERENCES}

1. Haritha C, Reddy AG, ReddyYR, AnjaneyuluY, RaoTM, Kumar BA, et al. Evaluation of protective action of fenugreek, insulin and glimepiride and their combination in diabetic Sprague Dawley rats. J Nat Sc Biol Med. 2013;4(1):207-12. https:// doi.org/10.4103/0976-9668.107292 ; PMid:23633864 PMCid:PMC3633279.
2. El-AbharHS, Schaalan MF. Phytotherapy in diabetes: Review on potential mechanistic perspectives. World J Diabetes. 2014;5(2):176-97. PMid:24748931 PMCid:PMC3990312.

3. Mishkinsky J, Joseph B, Sulman F. Hypoglycaemic effect of trigonelline. Lancet. 1967;290(7529):1311-2. https://doi.org/10.1016/S0140-6736(67)90428-X.

4. Hillaire-Buys D, Petit P, Manteghetti M, Baissac Y, Sauvaire Y, Ribes A. Recently identified substance extracted from fenugreek seeds stimulates insulin secretion in rat. Diabetologia. 1993;36:A119.

5. Sauvaire Y, Petit P, Broca C. 4-hydroxyisoleucine: a novel amino acid potentiator of insulin secretion. Diabetes Mag.1998;47(2):206-10. https://doi.org/10.2337/ diab.47.2.206

6. Madar Z. Fenugreek (Trigonella foenum graecum) as a means of reducing postprandial glucose level in diabetic rats. Nutr Rep Int. 1984;29:1267-73.

7. Shani J, Goldschmied A, Ahronson Z, Sulman FG. Hypoglycaemic effect of Trigonella foenum graecum and Lupinus termis (leguminosae) seeds and their major alkaloids in alloxan diabetic and normal rats. Arch Int Pharmacodyn Ther. 1974;210(1):27-36.

8. Ghosal S, Srivastava RS, Chatter DC, Dutta SK. Extractives of Trigonella - Fenugreekine, a new steroidal sapogenins -peptide ester of Trigonella foenum graecum. Phytochem. 19741;3:2247-51.

9. Handa SS, Khanuja SPS, Longo G, Rakesh DD. Extraction technologies for medicinal and aromatic plants. In; An overview of extraction techniques for medicinal and aromatic plants. International Centre for Science and High Technology: Italy; 2008.p.21-52.

10. Chopra S, Motwani SK, labal Z, Ahmad FJ, Khar RK. Simple, sensitive, selective and validated spectrophotometric methods for the estimation of a biomarker trigonelline from polyherbal gels. Mol Biomol Spectrosc. 2007;68(3):516-22. https://doi.org/10.1016/j.saa.2006.12.021; PMid:17336136.

11. Powder flow. In: United States Pharmacopoeia, 30th ed, NF 25: The Official Standard of Compendia; 2007.p.1174.

12. Bulk density and tapped Density. In: United States Pharmacopoeia. 30th ed. NF 25: The Official Standard of Compendia: 2007.p.1186. PMCid:PMC2292500.

13. Van Hostetler. Hard capsules. In: Leon Lachman, Herbert AL, Joseph LK, Editors. The theory and practice of industrial pharmacy. Bombay: Varghese Publishing House. 1991.p.374.

14. Rakieten N, Rakieten ML, Nadkarni MV. Studies on the diabetogenic action of streptozotocin. Cancer Chem other Rep. 1963;29:91-102.

15. Priya $V$, Jananie RK, Vijayalakshmi K. Anti-diabetic effect of Trigonella foenum graecum in diabetic rats-an in vivo study. Int J Pharm Sci. 2012;3(2):204-14

16. Vogel HG, VogelWH. Drug discovery and evaluation. 2nd ed. New York: SpringerVerlag Berlin Heidelberg; 2002. p.695. https://doi.org/10.1007/3-540-29837-1; PMid:12460729.

17. Gupta A, GuptaR, Lal B. Effect of Trigonella foenum graecum (fenugreek) seeds on glycaemic control and insulin resistance in type 2 diabetes mellitus: a double-blind placebo controlled study. J Assoc Physicians. India. 2001;49:1057-61. PMid:11868855

18. Vijaya SK, Ravishanker D, Kumar A, Annapurna MM. Binding efficacy of Trigonella foenum-graecum seed powder: tablet excipients. Drug Invent Today. 2012; 4(12):644-8.

19. Senthil V, Sripreethi D. Formulation and evaluation of paracetamol suspension from Trigonella foenum graecum mucilage. J Adv Pharm Educ Res. 2011; 1(5):225-33

20. Gowthamrajan K, Kulkarni GT, Muthukumar A. Evaluation of fenugreek mucilage as gelling agent. Int J PharmExp. 2002;3:16-9

21. Ali N, Hossein N, Afagh K, Tarifeh SHV, Ford JL. An in vitro evaluation of fenugreek mucilage as a potential excipient for oral controlled-release matrix tablet. Drug Dev Ind Pharm. 2008;34(3):323-9. https://doi.org/10.1080/03639040701662594; PMid:18363148. 